\title{
Should we or should we not? A phenomenological approach to understand health professionals' perception about adding information on stair climbing to physical activity questionnaires
}

\author{
Gloria Alva ${ }^{1}$, Pragnya Ravichandran ${ }^{2}$, Sundar Kumar Veluswamy ${ }^{3 *}$ \\ ${ }^{1}$ Lecturer, ${ }^{2}$ Postgraduate Student, ${ }^{3}$ Assistant Professor, Dept. of Physiotherapy, M S Ramaiah Medical College, Bengaluru, Karnataka, \\ India
}

\section{*Corresponding Author: Sundar Kumar Veluswamy}

Email: sundark94@gmail.com

\begin{abstract}
Introduction: Stair climbing being one of the most common suggestions as part of physical activity (PA) promotion, adding information regarding stair climbing as part of PA assessment could be beneficial but not usually done. This study was aimed at understanding the perception of health professionals about the need, feasibility, advantages and disadvantages of including stair climbing information as part of physical activity questionnaire.

Materials and Methods: Eight health professionals from departments of physiotherapy and community medicine were shortlisted based on their domain expertise and experience. Using a content analysis framework, in-depth semi-structured interviews were conducted among health professionals who consented to participate. The interviews were conducted at a mutually convenient time and audio recorded. Data coders performed verbatim transcription of the interviews to MS word for content analysis and themes were derived from the transcription by the investigators.

Results: All participants opined that stair climbing can be beneficial form of PA promotion and seven felt adding information about stair climbing is useful. Advantages reported by participants include capturing all activities of an individual, stair climbing being a part of regular PA and capturing calories burnt in stair climbing. Majority of dwellings not having stairs, less opportunity to climb stairs in urban areas due to presence of elevators, difficulty for individuals with pain/weakness and recall bias were mentioned as limitations. Most suggested adding a question to ascertain if they climb stairs as part of their routine before proceeding to ask about frequency.

Conclusion: Adding stair climbing information to PA questionnaires is needed but questions about its relevance in large population exist.
\end{abstract}

Keywords: Physical activity, Stair climbing, Physical activity questionnaire, Physical activity Promotion, Exercise.

\section{Introduction}

The link between lack of exercise and ill health is an acknowledged fact by health professionals worldwide. ${ }^{1,2}$ Lower levels of cardiopulmonary fitness is often associated with increased risk of developing non-communicable diseases (NCDs) thereby increasing the risk of morbidity and mortality. ${ }^{2-4}$ This was initially identified in a ground breaking discovery by Jerry Morris. ${ }^{5}$ His research among London bus drivers and conductors gave the initial leads of relationship between levels of Physical Activity (PA) and heart disease in the early 1950s. ${ }^{5}$ The health and fitness benefits linked with short, intermittent bouts of moderate to vigorous exercise have known to an influence in preventing cardiovascular disease risk and obesity. ${ }^{6}$

Regular stair climbing provides opportunities for performing moderate to vigorous intensity physical activity and is considered as a useful mode of exercise to improve cardiorespiratory fitness. ${ }^{7-9}$ It's a simple activity that most people often perform on daily basis and is considered to have a MET value above four. ${ }^{10}$ Despite being an activity of daily routine, stair climbing is not assessed as part of current PA questionnaires. ${ }^{11}$

Incidental PA represents a domain that is not planned and usually is the result of daily activities at work, at home, or during transport. ${ }^{12}$ Global Physical Activity Questionnaire (GPAQ) is an epidemiological physical activity questionnaire developed by World Health Organisation to assess physical activity. ${ }^{13}$ It assesses physical activity in work (occupational and domestic), transportation and leisure time domains but no information on stair climbing are taken in any of the domains. Apart from GPAQ there exist other questionnaires which assess PA, but like GPAQ they too do not include information on stair climbing. ${ }^{14}$ Therefore, stair climbing being one of the most common suggestions as part of PA promotion, details about its participation and frequency could be beneficial to be included as part of PA assessment.

Adding details about stair climbing to an existing epidemiological PA questionnaire would probably give an understanding of total physical activity performed. The proceeding cross-sectional study to the one described in this paper by our team showed that adding stair climbing information to an existing epidemiological questionnaire made a significant difference to total PA.

\section{Statement of problem}

Health professionals involved in health promotion and fitness industry routinely use questionnaires for the assessment for PA. Despite being an activity that can be incorporated into everyday life and having numerous health benefits, information on stair climbing is not currently being evaluated as part of routine physical activity assessment. Understanding the health professionals' perception of assessing stairclimbing as part of PA assessment may provide insights into current practice. It may also offer opportunities to understand barriers to assessing stair climbing information as part of PA assessment. This study was therefore planned to assess health professionals' perception about adding information on stair climbing to PA questionnaires. 


\section{Materials and Methods}

To address the objectives of the study, a qualitative research design using a phenomenological approach was used. ${ }^{15} \mathrm{~A}$ semi-structured in-depth interview method was adopted. The interview focused on health professionals' awareness about importance of PA, its assessment methods and relevance of adding stair climbing information to PA questionnaires. Potential participants were approached by trained research staff, explained about the need and purpose of the study, and the process of audio recording the interview. The interview was conducted at a mutually convenient time and in a silent ambience after the participants' provided written informed consent to participate.

\section{Participants}

Eight participants were recruited based on their subject expertise. Participants were selected from specialities of Community Medicine and Physiotherapy professionals working towards health promotion and fitness. The selected participants had prior knowledge and experience in health and PA promotion.

\section{Data collection}

Using a content analysis framework and in accordance with consolidated criteria for reporting qualitative studies guidelines, in-depth semi-structured interviews were conducted. ${ }^{16-17}$ Interview covered the domains of importance of PA and its assessment, health promotion strategies and value of stair climbing as PA assessment and were audio recorded. The interviewer was trained in qualitative research methods and during the interview prompts were provided as needed. The trained research staff performed verbatim transcription of the interviews which was then coded for analysis.

\section{Results}

Eight participants were shortlisted from the employees of the Institution and all provided written informed consent to participate in the study. Five participants were physiotherapists and the remaining three were community medicine physicians. The expertise of the participants ranged from being young clinicians practicing health promotion to experienced researchers involved in prevention and control of non-communicable diseases. Each interview lasted between 15-30 minutes and provided ample opportunities for the participants to express their views.

Data processing and analysis: All interviews were analysed using content analysis framework by three data coders. Audio records of the interviews were transcribed and processed for information. Codes were created for each new idea and themes that were found to be conceptually similar in nature or related in meaning. The analysis by the data coders derived 3 main themes from the interviews.

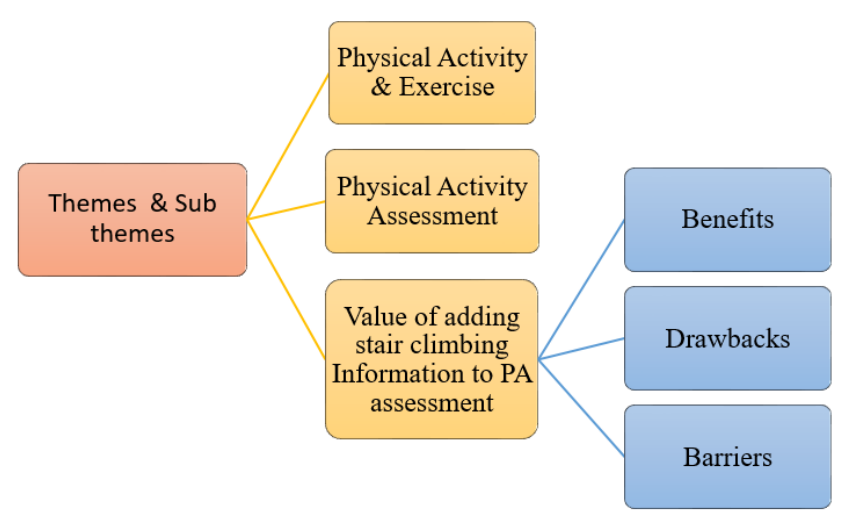

Fig. 1: Themes and the sub themes derived from the in-depth interviews

\section{Physical activity and exercise}

Knowledge and importance of everyday PA was evident among the participants who were interviewed. Nearly everyone agreed that there exists a difference between exercise and PA. Few explained exercise as being more structured while PA would be any bodily movements. Furthermore, a few explained PA as being over and above the regular day to day activities. Fewer participants were of the opinion on considering exercise as one of the components of total PA. The health benefits associated with being physically active such as its effect on the cardiopulmonary function, reducing the risks of CVD, obesity, cancer, and lower risks of having non-communicable diseases was identified by most participants.

From the interviews, it was evident that various strategies of promoting health through daily activities are needed. Incorporation of various health promotion strategies were also discussed with the participants during the interview and numerous ideas ranging from school health program to safe walk ways to encourage activity were suggested. Most participants were aware of the standard norms on being physically active for healthy living. Physiotherapy professionals were aware and well-informed about PA guidelines and current recommended levels of PA for achieving health benefits.

When interviewed about the use of stair climbing as a promotional strategy most participants opined it to be a good way to encourage people lead a healthy lifestyle. It was perceived that availability of the stairs in urban setup would make it an economical and feasible option for health promotion.

\section{Physical activity assessment}

All participants indicated about the utility of questionnaires as a PA assessment tool. Participants were also aware of wearable fitness monitors and pedometers as an option to assess PA. Knowledge on criterion measures of PA assessment such as indirect calorimetry, doubly labelled water was limited among about $50 \%$ of participants. The need for assessing PA under various domains such as work, transport, leisure, was considered important with the 
reasoning that it would provide a holistic information about an individual's PA level.

\section{Value of adding stair climbing Information to PA assessment}

All but one participant opined that stair climbing should be assessed as part of PA assessment. Capturing all activities that an individual performs on a typical day, knowing how many stairs individuals climb to use it as a PA promotion strategy and opportunity to estimate calories burnt in stair climbing were reported as benefits of adding stair climb information to PA assessment.

Two concerns emerged as barriers to adding information on stairclimbing. Stair climbing was identified as an easy physical activity recommendation within the typical urban workplace as a health enhancing activity. Some participants were of the view that majority of place may provide less opportunity or motivation to climb stairs in urban areas either due to non-availability of stairs in smaller buildings or presence of elevators in large buildings. Participants also indicated about lesser opportunities to use stairs in rural environments. It was pointed out that assessment of stair climbing in rural areas would not provide any significant gains in terms of PA assessment.

Difficulty in using stairs in individuals with pain and weakness were identified as drawbacks of adding information on stair climbing. Experienced researchers also expressed concerns with regard to recall bias and scope for under or overestimation of number of flights climbed by participants. This bias would lead to inaccurate estimation of PA levels over and above the inaccuracies that normally exists in PA questionnaires. Suggestions were made that a screening question could be included to ascertain if they climb stairs as part of their routine and if they answered in the affirmative, then questions regarding the frequency and duration of stair climbing could be considered.

\section{Discussion}

A recent publication from the on-going Harvad Alumni Health Study Cohort indicated that self-reported stair climbing was associated with all-cause and cardiovascular mortality. ${ }^{18}$ These findings are significant because they represent hard end points and not intermediaries or risk factors. Past research clearly indicates the health benefits of stair climbing and its utility as a means of health promotion strategy. ${ }^{7-9,18}$ Given the importance and benefits of stair climbing, it is not clear why this activity has not been considered as a component of PA assessment. The past PA guidelines had a recommendation of including moderate and vigorous intensity PA that were performed for a minimum of 10 minutes at a stretch. ${ }^{19}$ Since stair climbing as part of routine is usually a short duration activity lasting less than 23 minutes, it is possible that the evidence base precluded including information on stair climbing. The PA guidelines for Americans has been revised in 2018 and the current guidelines, for health benefits, do not emphasis on the 10minute cut-off that was mandated in previous guidelines. ${ }^{20}$ This revision is based on the research evidence that any amount of moderate-to-vigorous physical activity counts toward meeting the target range.

In this context, the views expressed by health professionals in our quality analysis assume importance because they represent a willingness and support to include stair climbing information as part of PA assessment. Though the number of health professionals interviewed were small, the views expressed were similar and were in agreement with previous pilot work, that included views of four health professionals. ${ }^{14}$

Though the barriers and drawbacks expressed by the participants are valid, they need to be viewed in light of the following details. Recall bias and under/over estimation is not unique to stair climbing questions but is a common concern for assessment of all domains of PA. In comparison to criterion measures, questionnaires have low validity as a measure of energy expenditure but have good discriminative validity and are considered a reliable tool for screening and assessment of PA. ${ }^{13}$ They also use generalisations such as attributing 4 METs for moderate intensity PA and 8 METs for vigorous intensity PA. ${ }^{19}$ In a similar manner, the 2011 PA compendium provides standardised MET values for stair climbing and these can be used to quantify the volume of PA obtained from stair climbing. ${ }^{10}$

Concerns were also raised about people with pain or weakness not being able to climb stairs. On similar lines, experts opined that assessing stair climbing may not add much value in environments that do not have stairs. The argument that India is a large country with majority of the population living in villages and hence adding stair climbing information may not add value at a population seems valid. But this needs to be seen in the same context as including vigorous intensity PA in assessment. Majority of individuals do not engage in vigorous intensity PA as part their work or leisure but its assessment as a dimension of PA is considered important and meaningful. On similar lines, stair climbing assessment of could be considered important and meaningful for participants who engage in the activity.

\section{Limitations}

This study included eight participants from one healthcare institution. Including more professionals from a larger cohort could have added more information about the views in diverse setting. The original research proposal was designed to assess the impact of adding stair climbing information to a PA questionnaire within a healthcare institution using a mixed method design. This limited the ability to recruit more participants for the qualitative study from other settings.

\section{Conclusion}

Among the eight professionals interviewed, there seemed to be agreement regarding the utility and benefits of adding stair climbing information to PA questionnaires.

\section{Funding}

This work was funded a faculty research grant from Rajiv Gandhi University of Health Sciences, Karnataka (Ref No. 15T001; PT-362, 2015-2016) 


\section{Acknowledgement}

The authors acknowledge the contribution of Dr. Abraham Samuel Babu, Associate Professor, Manipal College of Health Professions, MAHE for his contribution in the initial planning of this research.

\section{Conflict of interest}

None.

\section{References}

1. Booth FW, Roberts CK, Laye MJ. Lack of exercise is a major cause of chronic diseases. Compr Physiol 2012;2:1143-1211.

2. Nystoriak MA, Bhatnagar A. Cardiovascular Effects and Benefits of Exercise. Front Cardiovasc Med 2018;5:135.

3. Sheikholeslami S, Ghanbarian A, Azizi F. The Impact of Physical Activity on Non-communicable Diseases: Findings from 20 Years of the Tehran Lipid and Glucose Study. Int J Endocrinol Metab 2018;16(4 Suppl):e84740.

4. Lee IM, Shiroma EJ, Lobelo F, Puska P, Blair SN, Katzmarzyk $\mathrm{PT}$, et al. Effect of physical inactivity on major noncommunicable diseases worldwide: An analysis of burden of disease and life expectancy. Lancet 2012;380:219-29.

5. Morris JN, Heady JA, Raffle PA, Roberts CG, Parks JW. Coronary heart-disease and physical activity of work. Lancet 1953;262(6796):1111-20.

6. Murabito JM, Pedley A, Massaro JM. Moderate-to-vigorous physical activity with accelerometry is associated with visceral adipose tissue in adults. J Am Heart Assoc 2015;4(3):e001379.

7. Teh KC, Aziz AR. Heart rate, oxygen uptake, and energy cost of ascending and descending the stairs. Med Sci Sports Exerc. 2002;34:695-9.

8. Kennedy RA, Boreham CA, Murphy MH. Evaluating the effects of a low volume stair climbing programme on measures of health-related fitness in sedentary office workers. $J$ Sports Sci Med 2007;6:448-54.

9. Meyer P, Kayser B, Kossovsky MP. Stairs instead of elevators at workplace: cardioprotective effects of a pragmatic intervention. Eur J Cardiovasc Prev Rehabil 2010;17:569-75.

10. Ainsworth BE, Haskell WL, Herrmann SD. 2011 Compendium of Physical Activities: A second update of codes and MET values. Med Sci Sports Exerc 2011;43:1575-81.

11. Strath SJ, Kaminsky LA, Ainsworth BE. American Heart Association Physical Activity Committee of the Council on Lifestyle and Cardiometabolic Health and Cardiovascular, Exercise, Cardiac Rehabilitation and Prevention Committee of the Council on Clinical Cardiology, and Council. Guide to the assessment of physical activity: Clinical and research applications: a scientific statement from the American Heart Association. Circ 2013;128:2259-79.
12. Ziviani J, Scott J, Wadley D. Walking to school: incidental physical activity in the daily occupations of Australian children. Occup Therapy Int 2004;11:1.

13. Bull FC, Maslin TS, Armstrong T. Global physical activity questionnaire (GPAQ): nine country reliability and validity study. J Phys Act Health 2009;6:790-804.

14. Veluswamy SK, Maiya AG, Babu AS. PW228 Should Physical Activity Questionnaires Include Information on Stair Climbing? - A Mixed Methods Pilot Study. Global Heart 2014;9(1):e303-4.

15. Pietkiewicz I, Smith JA. A practical guide to using interpretative phenomenological analysis in qualitative research psychology. Psychological journal. 2014;20:7-14.

16. O'Brien BC, Harris IB, Beckman TJ, Reed DA, Cook DA. Standards for reporting qualitative research: a synthesis of recommendations. Acad Med 2014;89:1245-51.

17. Tong A, Sainsbury P, Craig J. Consolidated criteria for reporting qualitative research (COREQ): a 32-item checklist for interviews and focus groups. Int J Qual Health Care 2007;19:349-57.

18. Rey-Lopez JP, Stamatakis E, Mackey M. Associations of selfreported stair climbing with all-cause and cardiovascular mortality: The Harvard Alumni Health Study. Prev Med Rep 2019;15:100938.

19. World Health Organization. Global recommendations on physical activity for health. World Health Organization; 2010.

20. Piercy KL, Troiano RP, Ballard RM. The Physical Activity Guidelines for Americans. JAMA 2018;320:2020-8.

How to cite this article: Alva G, Ravichandran $P$, Veluswamy SK. Should we or should we not? A phenomenological approach to understand health professionals' perception about adding information on stair climbing to physical activity questionnaires. J Soc Indian Physiother 2020;4(1):10-3. 